CANCER

\title{
Incidence and management of malignant digestive endocrine tumours in a well defined French population
}

\author{
C Lepage, A M Bouvier, J M Phelip, C Hatem, C Vernet, J Faivre
}

Gut 2004;53:549-553. doi: 10.1136/gut.2003.026401

See end of article for authors' affiliations .....................

Correspondence to: C Lepage, Registre Bourguignon des Cancers Digestifs (INSERM EPI 0106), Faculté de Médecine, BP 87900 21079 Dijon Cedex France; jean.faivre@ u-bourgogne.fr

Accepted for publication 28 October 2003
Background and aims: Little is known about the epidemiology of malignant digestive endocrine tumours. The aim of this study was to report on their incidence and management in a well defined population. Methods: Data were obtained from the population based Digestive Cancer Registry of Burgundy (France) over a 24 year period. Incidence rates were calculated by sex, age groups, and period of diagnosis. Treatment and stage at diagnosis were also investigated. Prognosis was determined using crude and relative survival rates. A multivariate relative survival analysis was performed.

Results: Between 1976 and 1999, 229 cases were recorded. Age standardised incidence rates were $0.76 / 100000$ for men and $0.50 / 100000$ for women. They increased over time in both sexes. The resectability rate was $74.1 \%$. Among recorded cases, $26.6 \%$ did not extend beyond the organ, $20 \%$ had lymph node metastases, and $53.3 \%$ had visceral metastases or were unresectable. There was no improvement in the resection rate or in the stage at diagnosis over the study period. The overall relative survival rate was $66.9 \%$ at one year, $50.4 \%$ at five years, and $40.6 \%$ at 10 years. Stage at diagnosis, age at diagnosis, and subsite were independent significant prognostic factors.

Conclusions: Although their incidence is increasing, malignant digestive endocrine tumours remain a rare cancer, representing $1 \%$ of digestive cancers. Stage at diagnosis and prognosis at a population level are worse than those reported in hospital series. In the short term, new therapeutic possibilities represent the best way to improve their prognosis.
M alignant digestive endocrine tumours (MDET) have received little attention from epidemiologists. Information on their incidence is limited. This may be due to the fact that it is a rare condition and that the heterogeneity of this group of cancers arising from diverse sites have limited the study of their epidemiological characteristics. Moreover, increasing clinical and biological knowledge has led to a change in classification over time that makes studies difficult. The new histological typing of endocrine cancers of the International Histological Classification of Tumours ${ }^{1}$ pool digestive carcinoids and pancreatic endocrine tumours and separate them from endocrine-exocrine cancers and endocrine cancers developed within the limits of family pathology (multiple endocrine neoplasia type I and Recklinghausen syndrome). Because of the rarity of MDET, there is also a paucity of reports on their management and prognosis. Furthermore, available data are provided by specialised centres and as such cannot be used as reference because of unavoidable selection bias. Population based studies recording all cases in a well defined population represent the only way to assess the epidemiological characteristics and management of these cancers. Such studies are rare because they require accurate and detailed data collection which is seldom done systematically in cancer registries. Thus the objective of this study was to report the incidence, treatment, stage at diagnosis, and prognosis of MDET in a well defined French population.

\section{PATIENTS AND METHODS \\ Patients}

A population based cancer registry records all digestive tract cancers in the resident population of two administrative areas in Burgundy, France. These areas have a population of 1050000 according to the 1999 census. Cancer registration began in 1976 in one area, and in 1982 in the other. Information is actively collected by the cancer registry staff from multiple sources: pathology laboratories, university hospitals, local hospitals, private surgeons, oncologists and gastroenterologists, general practitioners, and monthly reviews of death certificates. Patients treated outside the area are identified through the National Health Service. The quality and completeness of the registry is certified every four years by an audit of the National Institute for Health and Medical Research and of the National Public Health Institute.

Because of the involvement of the entire medical profession, we assumed that nearly all newly diagnosed cases were recorded. All registered cancers with malignant endocrine morphology according to the International Classification of Diseases for oncology (ICD-O 2) were included ${ }^{2}$ : neuroendocrine carcinoma $(8246 / 3)$, insulinoma $(8151 / 3)$, gastrinoma $(8153 / 3)$, vipoma $(8155 / 3)$, glucagonoma $(8152 / 3)$, carcinoid $(8240 / 3)$, and pancreatic insular carcinoma (8150/3). Pathology reports were reviewed in order to classify tumours according to the WHO classification. ${ }^{1}$ Tumours showed signs of malignancy invasion of contiguous structures or documented metastasis in $75 \%$ of cases. In the others cases, following the WHO recommendation, ${ }^{1}$ gut tumours had to be invading the muscularis propria or beyond and to be more than $1 \mathrm{~cm}$ in diameter in the small intestine and in the large bowel. Pancreatic tumours had to be more than $3 \mathrm{~cm}$ in diameter to be being classified as MDET. The Ki67 proliferation index was not available for the oldest records.

Patients were divided into three age groups: $41.1 \%$ were $<65$ years, $31.7 \%$ were $65-74$ years, and $27.2 \%$ were $\geqslant 75$ years. Cancer site was classified according to the ICD-O 2 classification. ${ }^{2}$ Cancer extension at the time of diagnosis was classified following the TNM classification. ${ }^{3}$ Three stages were specified: stage I, resected cancers without

Abbreviations: MDET, malignant digestive endocrine tumours 
lymph node or visceral metastasis (T2-4 NO M0 for small and large bowel localisation, T2-T3 N0 M0 for pancreatic localisation); stage II, resected cancers with lymph node metastasis (Nl-2 M0); stage III, cancers with visceral metastasis ( $\mathrm{Ml}$ ) and/or locoregional extension making a complete surgical resection impossible. Surgical treatment was classified as: resection for cure (macroscopic resection of all malignant tissue and no microscopic evidence of surgical marginal spread $(n=127))$, palliative resection $(n=44)$, bypass, or exploratory laparotomy $(\mathrm{n}=30)$. Medical treatment included chemotherapy $(\mathrm{n}=49)$ and/or radiotherapy $(n=12)$. Survival of patients was ascertained from death certificates, registrar of the place of residence, or from practitioners. Life status was known for 228 patients (99.5\%) in January 2002.

\section{Methods}

The population data used for calculating incidence rates were based on population estimates by interpolation of the results of the four censuses $(1975,1982,1990,1999)$. Incidence rates were calculated by sex, age, and period of diagnosis. For the purpose of comparison with other countries, rates have been standardised by the direct method using the world standard population. Standard deviations were computed. Time trends in incidence were analysed using four five year periods from 1976 to 1995 and one four year period (1996-1999). Time trends in incidence by sex were assessed using a Poisson regression. The association between categorical data was analysed using the $\chi^{2}$ test. To study trends in MDET management and prognosis, two periods were defined: 1976-1987 and 1988-1999.

Crude survival rates were calculated using the life table method. Survival curves were compared using the log rank test. Relative survival rates were also calculated, these being defined as the ratio of the observed survival rate in the cancer patients under study to the expected survival rate in a population of similar sex and age distribution, subject only to the mortality rates of the general population. It reflects the excess mortality in the cancer patient group relative to the background mortality. Relative survival rates were computed using the RELSURV program. ${ }^{4}$ A multivariate survival analysis was performed using a relative survival model with proportional hazard applied to the net survival by interval. The significance of the covariates was tested by the likelihood ratio test.

\section{RESULTS}

All gut carcinomas were well differentiated. Previously they corresponded to malignant carcinoids. Among pancreatic tumours, $86.0 \%$ were non-secreting carcinomas and $14 \%$ were well differentiated functioning endocrine. Our series did not contain any small cell tumours.

\section{Incidence by sex, age, and period of diagnosis}

The 229 endocrine carcinomas represented $0.8 \%$ of digestive cancers in men and $1.1 \%$ in women. Mean ages at diagnosis were, respectively, 67.0 (SD 3.1) years and 64.8 (SD $2.3)$ years $(p=0.38)$. The overall crude annual incidence rate was 1.15 per 100000 for men (SD 0.10) and 0.91 per 100000 for women (SD 0.09). The corresponding age standardised incidence rates were, respectively, 0.76 (SD 0.07) and 0.50 (SD 0.06) per 100000 . The sex ratio calculated on age standardised rates was 1.5 .

Figure 1 shows the age specific incidence curves of MDET in men and women. MDET incidence rates were low and similar in both sexes before the age of 40 years. Thereafter the incidence rates increased more rapidly in men than in women until 75 years in men and 65 years in women. Then

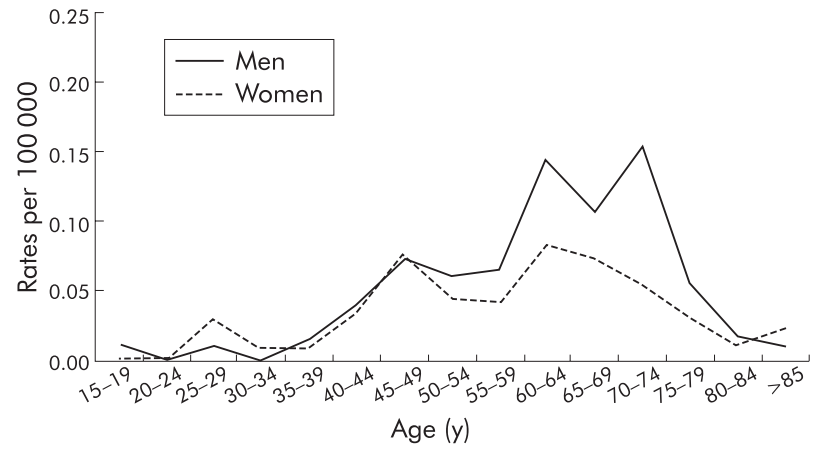

Figure 1 Age specific incidence rates of malignant digestive endocrine tumours in men and women.

the incidence rates dropped in the oldest age groups. Overall, $27.3 \%$ of cases were diagnosed after the age of 74 years.

Figure 2 shows trends over time in age standardised incidence rates by five year periods in men and women. Among men, incidence rates of MDET decreased slightly between the first and second study period $(p=0.89)$ and then increased throughout the last three study periods. In women, incidence rates increased progressively over time more rapidly than in men. However, the increase in incidence rates was significant in women and men $(p<0.001)$. During the first study period, age standardised incidence rates were 0.68/100 000 in men (SD 0.24) and 0.20/100 000 in women (SD 0.14). During the last study period they were, respectively, 1.01 (SD 0.18) and 0.90 (SD 0.17)/100 000.

\section{Incidence by site}

Among the 229 MDET, the small bowel was the most common site $(38.9 \%$ of cases), preceding large bowel localisations (27.1\%) and pancreatic localisations (20.5\%). The other sites were much less frequent: stomach $(6.1 \%)$, gall bladder $(2.2 \%)$, liver $(1.8 \%)$, oesophagus $(0.4 \%)$, peritoneum $(0.4 \%)$, and digestive localisation not otherwise stated (2.6\%). Appendix malignant endocrine tumours represented $8.1 \%$ of large bowel localisations. Age standardised incidence rates by site and sex are given in table 1 . There was a slight predominance in men for all sites. The sex ratio was slightly lower for small bowel localisations than for other sites. MDET represented $28.2 \%$ of small bowel carcinomas, $0.04 \%$ of large bowel carcinomas, and $1.7 \%$ of pancreatic carcinomas. There was no significant change in the distribution of MDET sites over time. When comparing the first and second study periods, the proportion of small bowel localisations was, respectively, $47.3 \%$ and $36.2 \%$, of large bowel localisations $21.8 \%$ and $28.7 \%$, and of pancreatic localisations $20.0 \%$ and $20.7 \%(\mathrm{p}=0.49)$.

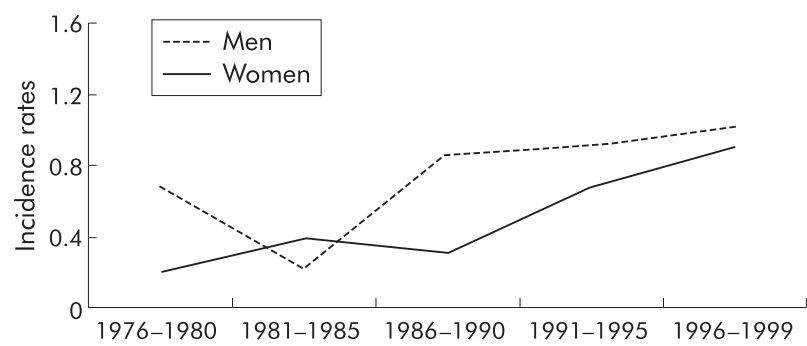

Figure 2 Age standardised incidence rates of malignant digestive endocrine tumours by sex and period. 
Table 1 Age standardised* incidence rates of malignant digestive endocrine tumours in men and women

\begin{tabular}{lllll}
\hline & $\mathbf{n}$ & Men & Women & Sex ratio \\
\hline Small bowel & 89 & 0.27 & 0.21 & 1.3 \\
Colon-rectum & 62 & 0.19 & 0.12 & 1.6 \\
Pancreas & 47 & 0.19 & 0.12 & 1.6 \\
Other sites & 31 & 0.11 & 0.07 & 1.6 \\
\hline
\end{tabular}

*World standard population per 100000 person years.

\section{Treatment and stage at diagnosis}

Resection for cure was performed in $54.5 \%$ of cases, palliative resection in $19.6 \%$, and exploratory laparotomy or bypass in $13.4 \%$. Overall, $12.5 \%$ of cases had no surgery. The proportion of resection for cure was $52.5 \%$ in men and $56.7 \%$ in women $(\mathrm{p}=0.271)$. It was $50.0 \%$ in patients less than 65 years old, $54.9 \%$ in the $65-74$ year age group, and $60.7 \%$ in those 75 years and over $(p=0.159)$. The rate of resection for cure varied with site of the tumour: it was higher for the large bowel $(63.1 \%)$ and small bowel $(62.9 \%)$ localisations than for pancreatic localisations $(38.3 \%)(\mathrm{p}<0.001)$. There was no significant variation in terms of treatment over time (table 2 ). The proportion of patients resected for cure was $61.8 \%$ during the $1976-87$ period and $52.1 \%$ during the $1988-99$ period $(\mathrm{p}=0.08)$.

No chemotherapy was performed between 1976 and 1983 , and then it was carried out in $22.7 \%$ of cases. During the second study period (1988-1999), chemotherapy was carried out after resection for cure in $21.9 \%$ of cases, after palliative surgery in $58.5 \%$ of cases, and among non-surgically treated patients in $19.5 \%$ of cases. Radiotherapy was performed in 12 cases.

Most MDET were already at a late stage at the time of diagnosis. Only $26.6 \%$ did not extend beyond the organ, $20.1 \%$ had lymph node metastases, and $53.3 \%$ had visceral metastases or were unresectable. Among metastatic cases, the proportion of patients with hepatic metastases was $59.7 \%$. Among these patients it was the only visceral metastasis localisation in $79.2 \%$ of cases. The other main metastatic sites were peritoneal carcinosis (23.2\% of cases), lung $(3.7 \%)$, skin $(2.4 \%)$, bone $(1.2 \%)$, and other sites $(9.7 \%)$. There was no significant change over time in the stage at diagnosis (table 2).

\section{Survival}

Overall five and 10 year observed survival rates were, respectively, $42.7 \%$ and $29.3 \%$, and the corresponding relative survival rates were $50.4 \%$ and $40.6 \%$. Table 3 shows the one and five year observed and relative survival rates for the studied variables. Period at diagnosis did not significantly influence the prognosis. Survival, both observed and relative (fig 3), was higher for women than for men. The effect of age was less marked after correction for risk of death other than the illness under study. Survival was related to the MDET site: it was better for MDET of the small intestine than for MDET of other sites. Treatment and stage at diagnosis were the most important determinants of survival. After curative resection, the five year relative survival rate was $72.6 \%$; it was $36.2 \%$ in the case of palliative resection and $17.7 \%$ in the case of other palliative treatments. Prognosis worsened with advancement of cancer stage. The five year relative survival rate varied from $80.1 \%$ in stage I to $35.8 \%$ in stage III.

As treatment and stage at diagnosis were closely correlated, they were not included together in the multivariate analysis. We made two successive multivariate survival models, including sex, age, site, period of diagnosis, and treatment in one of them, and stage at diagnosis in the other. The results are quite similar and only the second multivariate model is presented (table 4). For stage at diagnosis, the relative risk of death was 2.61 for stage II and 4.61 for stage III compared with stage I. Age and site were also significant prognostic factors independent of stage at diagnosis. In particular, large bowel MDET had a worse prognosis than small bowel MDET.

\section{DISCUSSION}

The epidemiology of MDET has not yet been clearly defined. This study is important as it is the first to report on the incidence and management of MDET at a population level. Incidence data were previously provided by a hospital based registry, including most diagnosed endocrine tumours in Northern Ireland. ${ }^{5}$ However, no distinction between benign and malignant neoplasms was made. Other population based studies have dealt only with carcinoid tumours. ${ }^{6-11}$ Again, no distinction between benign and malignant neoplasms was made in four reports. ${ }^{679}{ }^{10}$ In two studies, incidence rates of malignant carcinoids were provided using the US standard population. ${ }^{81}$ Another study comparable with ours, but limited to endocrine tumours of the gut, was performed in the Vaud area (Switzerland). ${ }^{12}$ Similar incidence rates were reported in both studies. Poor knowledge on the epidemiology of MDET can be explained by the rarity of these tumours, modifications over time in their histological definition, and by the fact that the definition of malignancy may appear

Table 2 Distribution of malignant digestive endocrine tumours (\%) by stage at diagnosis and treatment according to period at diagnosis

\begin{tabular}{llll}
\hline & $\begin{array}{l}1976-1987 \\
(\mathbf{n = 5 5 )}\end{array}$ & $\begin{array}{l}1988-1999 \\
(\mathbf{n = 1 7 4 )}\end{array}$ & p Value \\
\hline Stage & 20.0 & 24.3 & 0.810 \\
$\quad$ Limited to the organ & 21.8 & 20.7 & \\
$\quad$ Lymph node metastasis & 58.2 & 55.0 & \\
$\quad$ Visceral metastasis or unresected tumours & 61.8 & 52.1 & 0.070 \\
Treatment & 14.6 & 21.3 & \\
$\quad$ Resection for cure & & & \\
$\quad$ Palliative resection & 23.6 & 26.6 & \\
$\quad$ Other palliative treatments & & \\
\hline
\end{tabular}


Table 3 Crude and relative survival rates of malignant digestive endocrine tumours

\begin{tabular}{|c|c|c|c|c|c|c|c|}
\hline & \multirow[b]{2}{*}{$\mathbf{n}$} & \multicolumn{3}{|c|}{ Observed } & \multicolumn{3}{|l|}{ Relative } \\
\hline & & 1 year & 5 year & p Value & 1 year & 5 year & p Value \\
\hline Global & 229 & 65.5 & 42.7 & & 66.9 & 50.4 & \\
\hline \multicolumn{8}{|l|}{ Sex } \\
\hline Men & 123 & 62.8 & 38.1 & \multirow[t]{2}{*}{0.034} & 61.5 & 43.3 & \multirow[t]{2}{*}{0.025} \\
\hline Women & 106 & 68.0 & 47.5 & & 74.5 & 60.2 & \\
\hline \multicolumn{8}{|l|}{ Age (y) } \\
\hline$<65$ & 96 & 74.9 & 59.3 & \multirow[t]{3}{*}{0.0001} & 75.3 & 60.4 & \multirow[t]{3}{*}{0.050} \\
\hline $65-74$ & 71 & 61.5 & 34.7 & & 61.6 & 42.3 & \\
\hline$>75$ & 62 & 54.0 & 24.7 & & 56.8 & 36.5 & \\
\hline \multicolumn{8}{|l|}{ Site } \\
\hline Small bowel & 89 & 78.4 & 48.0 & \multirow[t]{4}{*}{0.291} & 78.4 & 66.5 & \multirow[t]{4}{*}{0.040} \\
\hline Large bowel & 62 & 57.6 & 36.4 & & 58.5 & 40.8 & \\
\hline Pancreas & 47 & 59.6 & 42.0 & & 61.3 & 44.2 & \\
\hline Other sites & 31 & 54.4 & 40.2 & & 58.5 & 40.8 & \\
\hline \multicolumn{8}{|l|}{ Period } \\
\hline 1976-1987 & 55 & 68.9 & 47.2 & \multirow[t]{2}{*}{0.151} & 71.2 & 55.8 & \multirow[t]{2}{*}{0.127} \\
\hline 1988-1999 & 174 & 63.9 & 39.9 & & 65.7 & 48.5 & \\
\hline \multicolumn{8}{|l|}{ Treatment } \\
\hline Resection for cure & 127 & 79.1 & 60.3 & \multirow[t]{3}{*}{$<0.0001$} & 84.5 & 72.6 & \multirow[t]{3}{*}{$<0.0001$} \\
\hline Palliative resection & 44 & 63.5 & 24.3 & & 57.6 & 36.2 & \\
\hline Other palliative treatment & 58 & 36.5 & 17.3 & & 39.0 & 17.7 & \\
\hline \multicolumn{8}{|l|}{ Stage } \\
\hline Limited to the organ & 57 & 82.0 & 64.9 & \multirow[t]{3}{*}{0.0001} & 88.2 & 80.1 & \multirow[t]{3}{*}{$<0.0001$} \\
\hline Lymph node metastasis & 47 & 69.6 & 49.9 & & 74.2 & 59.0 & \\
\hline Visceral metastasis or unresected tumours & 125 & 55.9 & 30.1 & & 55.9 & 35.8 & \\
\hline
\end{tabular}

ambiguous. This report indicates that cancer registry data, if precise morphology codes are available, can be used to study the epidemiology of MDET. One of the major problems faced by population based studies is determination of the completeness and reliability of the data. Our data included all cases diagnosed in a well defined French population during the period 1976-99, including untreated cases. All data were collected in a uniform fashion, regardless of the period of time or centre of diagnosis. Because of the multiplicity of information sources, we assume that nearly all newly diagnosed cases of MDET were recorded. Furthermore, information on treatment, stage at diagnosis, and survival were almost exhaustive. This study was therefore carried out without selection bias.

This study confirms that MDET are rare tumours, representing approximately $1 \%$ of all digestive cancers. They are characterised by a slight predominance in men. Mean age at diagnosis of MDET was slightly lower than age at diagnosis for other digestive cancers (70.5 years (SD 12.0) in this area). In hospital based statistics, age at diagnosis is generally lower

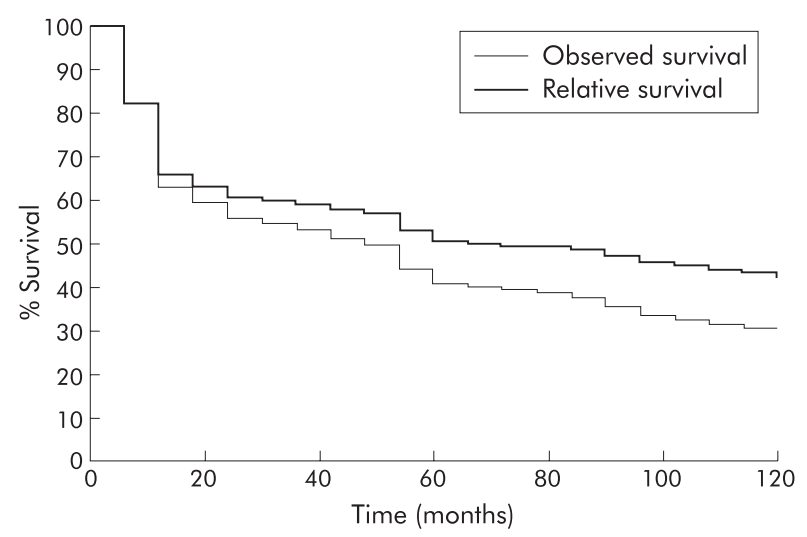

Figure 3 Observed and relative survival of malignant digestive endocrine tumours. than in our study, suggesting that the oldest patients are rarely seen in specialised centres. Levelling off of incidence rates in the oldest age groups can be attributed to less accurate diagnosis. The current study also showed an increase in incidence over time in both sexes. It is difficult to explain these trends. Improvement in diagnostic strategy or in diagnostic criteria is an improbable explanation for gut lesions. They may have a small effect on pancreatic tumours in relation to the increase in histologically verified cases. Furthermore, change in diagnostic criteria cannot explain the fact that incidence increased more rapidly in women than in men. Aetiological factors probably play a role but, because of the rarity of these tumours, they are not yet known. The small bowel is the most frequently occurring localisation for MDET. The predominance of these sites has already been noted by others. ${ }^{7}$ The large bowel and pancreas represent the two other main localisations. MDET of other sites were very rare. If carcinoid tumours of the appendix are relatively common neoplasms, they are usually benign. ${ }^{13}$ In more than half a century of practice at the Mayo Clinic, only two deaths related to metastatic appendiceal carcinoids were documented. Metastasis was never seen in patients with tumours less than $2 \mathrm{~cm}$. We confirm the rarity of MDET of the appendix. Only five cases were reported in our series (2.1\% of cases).

One interesting aspect of this study was to describe the management of MDET at a community level. It is well accepted that surgical excision of the lesion, when possible, is always preferable. In our population, slightly more than half of the cases were resected for cure. This rate was lower than that related to hospital based series which assess resectability rates varying between $80 \%$ and $90 \% .{ }^{14-16}$ This discrepancy is not surprising. Hospital data have been provided by specialised units and as such cannot be used as reference because of unavoidable selection bias. A less favourable therapeutic approach has already been reported in community based statistics for others cancers sites. The proportion of patients receiving chemotherapy has increased. It has been proven to have an antitumoral effect on metastases which seem lower in gut tumours than in pancreatic tumours. ${ }^{17-19}$ Nevertheless, the practice of chemotherapy is still relatively limited. It has not yet reached its full development at a 
Table 4 Multivariate relative survival analysis in malignant digestive endocrine tumours

\begin{tabular}{|c|c|c|c|}
\hline & Relative risk & $p$ Value & $95 \% \mathrm{Cl}$ \\
\hline \multicolumn{4}{|l|}{ Age (y) } \\
\hline$<65$ & 1.00 & 0.028 & \\
\hline $65-74$ & 1.79 & & $1.09-2.94$ \\
\hline$>75$ & 1.88 & & $1.06-3.34$ \\
\hline \multicolumn{4}{|l|}{ Site } \\
\hline Small bowel & 1.00 & 0.020 & \\
\hline Large bowel & 2.44 & & $1.35-4.44$ \\
\hline Pancreas & 1.84 & & $0.99-3.43$ \\
\hline Other sites & 1.94 & & $0.96-3.92$ \\
\hline \multicolumn{4}{|l|}{ Stage } \\
\hline Limited to the organ & 1.00 & $<0.001$ & \\
\hline Lymph node metastasis & 2.61 & & $0.98-6.98$ \\
\hline Visceral metastasis or unresected tumours & 4.61 & & $2.01-10.58$ \\
\hline
\end{tabular}

population level. There is wide variation in the proportion of advanced cases (metastatic or unresectable cases) in hospital based series. It varied between $35 \%{ }^{20}$ and $71 \% .{ }^{11}$ Our study indicates that stage at diagnosis is advanced when considering a general population. On average, $55 \%$ of patients had visceral metastases or unresectable tumours at the time of diagnosis. This study also indicated that there was no significant improvement in resection rate or in the stage at diagnosis of patients with MDET over the 24 years of the study, which is disappointing. Changes in diagnostic strategies, with the development of imaging techniques, did not contribute to improvement in the management of MDET.

The development of MDET is thought to be slow. If the prognosis is better than for other digestive cancers, except the large bowel, it is not as good as that suggested by hospital statistics. For example, in a French series of 82 malignant endocrine tumours of the duodenopancreatic area, the five year crude survival rate was $100 \%$ in the absence of liver metastasis and $40 \%$ in their presence. ${ }^{16}$ Population based statistics represent the only way of assessing the real survival rate of MDET patients. The prognosis of MDET not extending beyond the organ is comparable with that of other types of digestive cancers. Mainly, advanced MDET are less aggressive than other digestive cancers. In this situation, aggressive treatments are justified to further improve survival.

It is widely accepted that stage at diagnosis is the major prognosis factor..$^{21}$ Complete resection of the lesion, which is strongly correlated with stage at diagnosis, is also an important prognosis factor. ${ }^{16}$ MDET of the large bowel appear to behave like aggressive malignant neoplasms. Survival rates of patients with MDET of the large bowel are worse than those of patients with adenocarcinoma of the colon. ${ }^{1122}$ Survival rates similar to ours have been reported by the Connecticut tumour registry. ${ }^{8}$ The aggressiveness of these tumours is confirmed by the fact that the large bowel is a prognostic factor, independent of stage at diagnosis. Clinicians must take into account the severity of large bowel MDET. In the absence of improvement over time of stage at diagnosis and of resectability, it is not surprising that no significant improvement in the prognosis of MDET has been achieved over the 24 years of the study. Because of the lateness of symptoms, earlier diagnosis is an objective difficult to achieve. In the short term, more trials are needed to appreciate the benefits of adjuvant or palliative therapy for MDET. New approaches to the treatment of MDET also need to be found.

\section{Authors' affiliations}

C Lepage, A M Bouvier, J M Phelip, C Hatem, C Vernet, J Faivre, Faculté de Médecine, Registre Bourguignon des Cancers Digestifs (INSERM EPI 0106), Dijon, France

\section{REFERENCES}

1 Solcia E. Histological typing of endocrine tumours. International histological classification of tumours, 2nd edn. Berlin: Springer-Verlag, 2000.

2 ICD-02. International classification of diseases for oncology. Geneva: World Health Organisation, 1992.

3 Sobin LH, Wittekind C. TNM Classification of Malignant Tumors Union Internationale Contre le Cancer and the American Joint Committee on Cancer, 5th edn. New York: John Wiley and Sons, 1997.

4 Hédelin G. RELSURV 1.0, a program for relative survival. Strasbourg: Presse Universitaire, 1995

5 Watson RG, Johnston CF, O'Hare MM, et al. The frequency of gastrointestinal endocrine tumours in a well-defined population. Northern Ireland 19701985. Q J Med 1989;72:647-57.

6 Berge T, Linell F. Carcinoid tumours. Frequency in a defined population during a 12-year period. Acta Pathol Microbiol Scand A 1976:84:322-30.

7 Newton JN, Swerdlow AJ, dos Santos Silva IM, et al. The epidemiology of carcinoid tumours in England and Scotland. Br J Cancer 1994;70:939-42.

8 Ballantyne GH, Savoca PE, Flannery JT, et al. Incidence and mortality of carcinoids of the colon. Data from the Connecticut Tumor Registry. Cancer 1992:69:2400

9 Hemminki K, Li X. Incidence trends and risk factors of carcinoid tumors: a nationwide epidemiologic study from Sweden. Cancer 2001;92:2204-10.

10 Quaedvlieg PF, Visser O, Lamers CB, et al. Epidemiology and survival in patients with carcinoid disease in The Netherlands. An epidemiological study with 2391 patients. Ann Oncol 2001;12:1295-300.

11 Modlin IM, Lye KD, Kidd M. A 5-decade analysis of 13,715 carcinoid tumors. Cancer 2003;97:934-59.

12 Levi F, Randimbison L, Franceschi S, et al. Descriptive epidemiology of malignant carcinoids in the swiss canton of Vaud. Int J Cancer 1993:53:1036-7.

13 Moertel CG, Weiland LH, Nagorney DM, et al. Carcinoid tumor of the appendix: treatment and prognosis. N Engl J Med 1987;317:1699-701.

14 Corleto VD, Panzuto F, Falconi $M$, et al. Digestive neuroendocrine tumours: diagnosis and treatment in Italy. A survey by the Oncology Study Section of the Italian Society of Gastroenterology (SIGE). Dig Liver Dis 2001;33:217-21.

15 Shebani KO, Souba WW, Finkelstein DM, et al. Prognosis and survival in patients with gastrointestinal tract carcinoid tumors. Ann Surg 1999;229:815-21.

16 Madeira I, Terris B, Voss $M$, et al. Prognostic factors in patients with endocrine tumours of the duodenopancreatic area. Gut 1998:43:422-7.

17 Moertel CG. Karnofsky memorial lecture. An odyssey in the land of small tumors. J Clin Oncol 1987;5:1502-22.

18 Moertel CG, Lefkopoulo M, Lipsitz S, et al. Streptozocin-doxorubicin, streptozocin-fluorouracil or chlorozotocin in the treatment of advanced isletcell carcinoma. N Engl J Med 1992;326:519-23.

19 Broder LE, Carter SK. Pancreatic islet cell carcinoma. II. Results of therapy with streptozotocin in 52 patients. Ann Intern Med 1973;79:108-18.

20 Burke AP, Thomas RM, Elsayed AM, et al. Carcinoids of the jejunum and ileum: an immunohistochemical and clinicopathologic study of 167 cases. Cancer 1997;79: 1086-93.

21 Hochwald SN, Zee S, Conlon KC, et al. Prognostic factors in pancreatic endocrine neoplasms: an analysis of 136 cases with a proposal for low-grade and intermediate-grade groups. J Clin Oncol 2002;20:2633-42.

22 Faivre-Finn C, Bouvier A-M, Phelip J-M, et al. Colon cancer in France: evidence for improvement in management and survival. Gut 2002;51:60-4. 